\title{
Global Existence and Exponential Decay for a Dynamic Contact Problem of Thermoelastic Timoshenko Beam with Second Sound
}

\author{
Wenjun Liu *, Dongqin Chen and Biqing Zhu \\ College of Mathematics and Statistics, Nanjing University of Information Science and \\ Technology, Nanjing 210044, China; cdqlinsey@163.com (D.C.); brucechu@163.com (B.Z.) \\ * Correspondence: wjliu@nuist.edu.cn
}

In this paper, we study the global existence and exponential decay for a dynamic contact problem between a Timoshenko beam with second sound and two rigid obstacles, of which the heat flux is given by Cattaneo's law instead of the usual Fourier's law. The main difficulties arise from the irregular boundary terms, from the low regularity of the weak solution and from the weaker dissipative effects of heat conduction induced by Cattaneo's law. By considering related penalized problems, proving some a priori estimates and passing to the limit, we prove the global existence of the solutions. By considering the approximate framework, constructing some new functionals and applying the perturbed energy method, we obtain the exponential decay result for the approximate solution, and then prove the exponential decay rate to the original problem by utilizing the weak lower semicontinuity arguments.

Keywords: thermoelastic Timoshenko beam; global existence; exponential stability; second sound

AMS Subject Classification (2000): 74M15, 35B40, 93D20.

\section{Introduction}

In this paper, we investigate the mechanical behavior of thermoelastic Timoshenko homogeneous beam, of natural length $l$, which may come in contact with two rigid obstacles (see Figure 1). We denote by $\varphi=\varphi(x, t), \psi=\psi(x, t), \theta=\theta(x, t)$ and $q=q(x, t)$ the transverse displacement, angle, relative temperature and heat flow, respectively, we consider the following system:

$$
\begin{cases}\rho_{1} \varphi_{t t}(x, t)-k\left[\varphi_{x}(x, t)+\psi(x, t)\right]_{x}+\alpha \varphi_{t}(x, t)=0, & (x, t) \in(0, l) \times(0, T), \\ \rho_{2} \psi_{t t}(x, t)-b \psi_{x x}(x, t)+k\left[\varphi_{x}(x, t)+\psi(x, t)\right]-m \theta_{x}(x, t)=0, & (x, t) \in(0, l) \times(0, T), \\ \theta_{t}(x, t)+r q_{x}(x, t)-m \psi_{x t}(x, t)=0, & (x, t) \in(0, l) \times(0, T), \\ \tau q_{t}(x, t)+q(x, t)+r \theta_{x}(x, t)=0, & (x, t) \in(0, l) \times(0, T),\end{cases}
$$

with the initial conditions

$$
\begin{gathered}
\varphi(x, 0)=\varphi_{0}(x), \varphi_{t}(x, 0)=\varphi_{1}(x), \quad x \in[0, l], \\
\psi(x, 0)=\psi_{0}(x), \psi_{t}(x, 0)=\psi_{1}(x), \quad x \in[0, l], \\
\theta(x, 0)=\theta_{0}(x), q(x, 0)=q_{0}(x), \quad x \in[0, l]
\end{gathered}
$$


and

$$
\begin{gathered}
\varphi(0, t)=0, \psi(0, t)=0, q(0, t)=0, \quad t \in[0, T], \\
\psi_{x}(l, t)=0, \quad \theta(l, t)=0, \quad t \in[0, T],
\end{gathered}
$$

for some given functions $\varphi_{0}, \varphi_{1}, \psi_{0}, \psi_{1}, \theta_{0}, q_{0}$. The coefficients $\rho_{1}, \rho_{2}, b, k, \alpha, m$ and $\tau$ represent the mass density, the moment of mass inertia, the rigidity coefficient of cross section, the shear modulus of elasticity, the coefficient of the damping force, the coupling coefficient depending on the material properties and the thermal diffusivity, respectively, with $\rho_{1}, \rho_{2}, k, b, \tau, \alpha \in \mathbb{R}_{+}$and $m \in \mathbb{R} \backslash\{0\}$.

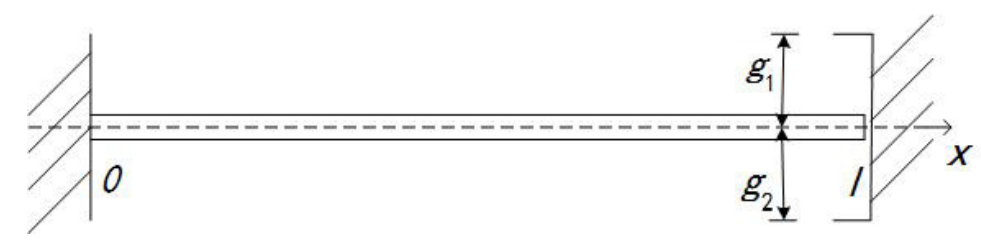

Figure 1: A thermoelastic Timoshenko beam and the tip at $x=l$ with clearance $g=g_{1}+g_{2}$.

The tip at $x=l$ is modeled with the Signorini non-penetration condition, see [20, 27]. In particular, the tip with gap $g$ is the asymmetrical so that $g=g_{1}+g_{2}$, where $g_{1}>0$ and $g_{2}>0$ are, respectively, the upper and lower clearances, when the system is at rest (see Figure 1). Then, the right end of the beam is assumed to move vertically only between two stops, namely

$$
-g_{2} \leq \varphi(l, t) \leq g_{1}, \quad t \in(0, T)
$$

We denote by $\sigma(t)$ the shear stress at $x=l$, i.e.,

$$
\sigma(t):=k\left[\varphi_{x}(l, t)+\psi(l, t)\right]
$$

We require that when there is no contact, namely $-g_{2}<\varphi(l, t)<g_{1}$, the right end is free and $\sigma(t)=0$. On the other hand, when $u(l, t)$ is in contact, namely $\varphi(l, t)=-g_{2}$ or $\varphi(l, t)=g_{1}$, the stress is opposite to the displacement: $\sigma(t) \geq 0$ if $\varphi(l, t)=-g_{2}$ and $\sigma(t) \leq 0$ if $\varphi(l, t)=g_{1}$. Accordingly, we prescribe

$$
-\sigma(t) \in \partial \mathcal{X}(\varphi(l, t)), \quad t \in[0, T],
$$

where $\partial \mathcal{X}$ denotes the subdifferential of the indicator function $\mathcal{X}$

$$
\mathcal{X}(\varphi)= \begin{cases}0, & \text { if }-g_{2}<\varphi<g_{1} \\ +\infty, & \text { otherwise }\end{cases}
$$

namely

$$
\partial \mathcal{X}(\varphi)= \begin{cases}(-\infty, 0], & \text { if } \varphi=-g_{2}, \\ 0, & \text { if }-g_{2}<\varphi<g_{1} \\ {[0,+\infty),} & \text { if } \varphi=g_{1}\end{cases}
$$


Many researchers got interested in studying the dynamics contact problems involving only a single displacement and/or a single variation of temperature, see for example $[2,3,20,21,27$, 36, 42]. Carlson [11] and Day [17] found that two or more materials may come in contact as a result of thermoelastic expansion or contraction in industrial processes. Copetti [14], Kuttler and Shillor [26] proposed the dynamic evolution of a thermoviscoelastic rod which may contact or impact a rigid or reactive obstacle, whereas the exponential energy decay rate for weak solutions of a thermoelastic rod, contacting a rigid obstacle, has been analyzed in [34]. Copetti [15] proved existence and uniqueness results and proposed finite element approximations in space with backward Euler discretization in time for a contact problem in generalized thermoelasticity under the theory of thermoelasticity proposed by Green and Lindsay [23]. Berti and Naso [9] considered the existence and longtime behavior of solutions for a dynamic contact problem between a nonlinear viscoelastic beam and two rigid obstacles. Afterward, thermal effects have been also taken into account in $[6,8]$, where Berti et al. proved the existence and uniqueness of the solution as well as the exponential decay of the related energy.

Timoshenko beam with thermal contribution have been investigated by many authors and some results related to global existence and decay properties have been obtained, see for example $[12,13,18,19,22,24,28,31,33,41,44]$. For the case of nonlinear internal frictional damping and without thermal effects, we refer the readers to Boussouira [1], Rivera and Racke [35], Raposo et al. [40] and Soufyane [43]. The boundary stabilization and boundary control have been studied in $[25,46]$ (see also references therein). Arantes and Rivera in [4] proved that the energy associated with the thermoelastic Timoshenko beam system decays exponentially as time goes to infinity. Meanwhile, a great number of researchers have devoted considerable amount time studying Timoshenko beam with contact problems. For instances, in [5], Araruna et al. showed the existence of solutions and the exponential stability of the energy for a contact problem associated with an elastic Timoshenko beam and a rigid obstacle under the assumption of a dissipative boundary feedback. Berti et al. [7] proved global existence in time of solutions and exponential decay for a dynamic contact problem between a Timoshenko beam and two rigid obstacles. In [16], well-posedness and fully discrete approximations for a dynamic contact problem between a viscoelastic Timoshenko beam and a deformable obstacle was analyzed.

In the above-mentioned result of Berti et al. [7], the heat dissipation is given through Fourier's law. As it is well known, by using the Fouriers law for the heat conduction, the thermal effect is propagated in an infinite speed in thermoelasticity. To overcome this physical paradox, many theories have been developed. Lord and Shulman [30] suggested that Fouriers law was replaced by Cattaneos law to describe the heat conduction, which transforms the classical thermoelastic system into the thermoelastic system with second sound, in which the thermal disturbance is propagated in a finite speed. Over the past decade, sevaral asymptotic behavior results have been obtained for the thermoelasticity system with second sound $([10,29,32,37,38,39,45])$. Berti et al. [8] investigated a dynamic contact problem describing the mechanical and thermal evolution of a damped extensible thermoviscoelastic beam under the Cattaneo law. 
Motivated by these results, the aim of the present paper is to establish a global in time existence result to problem (1.1)-(1.6) and analyze its longtime behavior. In particular, we prove that the system possesses an energy decaying exponentially as time goes to infinity. Problem (1.1)(1.6) can be regarded as an extension and improvement of Berti et al. [7] to the thermoelastic Timoshenko beam with second sound. It has been shown in [22] that the dissipative effects of heat conduction induced by Cattaneo's law are usually weaker than those induced by Fourier's law, and the coupling via Cattaneo's law may cause loss of the exponential decay usually obtained in the case of coupling via Fouriers law. The main difficulties also arise from the irregular boundary terms induced by the constraint (1.6) and from the low regularity of the weak solution. In order to prove the global existence result, we consider an approximate version of problem (1.1)-(1.6) by introducing a normal compliance condition as regularization of the Signorini condition (1.6). We first prove a well-posedness result for the penalized problem by means of a Faedo-Galerkin scheme, and then derive suitable a priori estimates and pass to the limit in the regularization parameter obtaining the existence of a solution to the original problem. In order to get the exponential decay result to problem (1.1)-(1.6), we consider the approximate framework. By introducing a suitable Lyapunov functional and using the multiplier method, we first obtain the exponential decay result for the approximate solution. Then, under weak lower semicontinuity arguments, we prove the exponential decay rate for a solution to the original problem.

The paper is organized as follows. In Section 2, a variational formulation of problem (1.1)-(1.6) has been introduced and the main results have been stated. In Section 3, we study the existence of a weak solution to problem (1.1)-(1.6). The exponential stability result is proved in Section 4.

\section{Main results}

To give a variational formulation of the problem, we introduce the following spaces:

$$
\begin{aligned}
& \mathcal{V}=\left\{f \in H^{1}(0, l): f(0)=0\right\} \\
& \mathcal{K}=\left\{\varphi \in \mathcal{V}:-g_{2} \leq \varphi(l) \leq g_{1}\right\} \\
& \mathcal{H}=\left\{f \in H^{1}(0, l): f(l)=0\right\}
\end{aligned}
$$

The initial data

$$
\left(\varphi_{0}, \psi_{0}, \theta_{0}, q_{0}\right) \in \mathcal{K} \times \mathcal{V} \times L^{2}(0, l) \times L^{2}(0, l), \quad\left(\varphi_{1}, \psi_{1}\right) \in\left[L^{2}(0, l)\right]^{2}
$$

We define

$$
\begin{aligned}
E(t):=E(t, \varphi, \psi, \theta, q)= & \frac{1}{2} \int_{0}^{l}\left[\rho_{1}\left|\varphi_{t}(x, t)\right|^{2}+\rho_{2}\left|\psi_{t}(x, t)\right|^{2}+|\theta(x, t)|^{2}+\tau|q(x, t)|^{2}\right. \\
& \left.+k\left|\varphi_{x}(x, t)+\psi(x, t)\right|^{2}+b\left|\psi_{x}(x, t)\right|^{2}\right] \mathrm{d} x
\end{aligned}
$$

as the energy associated with system (1.1)-(1.5). 
Definition 2.1 Let $\varphi_{0}, \psi_{0}, \theta_{0}, q_{0}, \varphi_{1}, \psi_{1}$ be given as in (2.1) and $0<T \leq \infty$. We say that $(\varphi, \psi, \theta, q)$ is a weak solution to problem (1.1)-(1.6) when

$$
\begin{aligned}
& \varphi \in W^{1, \infty}\left(0, T ; L^{2}(0, l)\right) \cap L^{\infty}(0, T ; \mathcal{K}), \\
& \psi \in W^{1, \infty}\left(0, T ; L^{2}(0, l)\right) \cap L^{\infty}(0, T ; \mathcal{V}), \\
& \theta \in L^{\infty}\left(0, T ; L^{2}(0, l)\right), \\
& q \in L^{\infty}\left(0, T ; L^{2}(0, l)\right),
\end{aligned}
$$

with initial data satisfying (1.2), the inequality

$$
\begin{aligned}
& \int_{0}^{T} \int_{0}^{l}\left\{-\rho_{1} \varphi_{t}(x, t)\left[\omega_{t}(x, t)-\varphi_{t}(x, t)\right]+k\left[\varphi_{x}(x, t)+\psi(x, t)\right]\left[\omega_{x}(x, t)-\varphi_{x}(x, t)\right]\right. \\
& \left.\quad+\alpha \varphi_{t}(x, t)[\omega(x, t)-\varphi(x, t)]\right\} \mathrm{d} x \mathrm{~d} t \geq \rho_{1} \int_{0}^{l} \varphi_{1}(x)\left[\omega(x, 0)-\varphi_{0}(x)\right] \mathrm{d} x,
\end{aligned}
$$

for every $\omega \in W^{1,1}\left(0, T ; L^{2}(0, l)\right) \cap L^{2}(0, T ; \mathcal{K})$ such that $\omega(\cdot, T)=\varphi(\cdot, T)$, and the equations

$$
\begin{aligned}
\int_{0}^{T} & \int_{0}^{l}\left\{-\rho_{2} \psi_{t}(x, t) \mathcal{X}_{t}(x, t)+b \psi_{x} \mathcal{X}_{x}(x, t)+k\left[\varphi_{x}(x, t)+\psi(x, t)\right] \mathcal{X}(x, t)\right. \\
& \left.+m \theta(x, t) \mathcal{X}_{x}(x, t)\right\} \mathrm{d} x \mathrm{~d} t=\rho_{2} \int_{0}^{l} \psi_{1}(x) \mathcal{X}(x, 0) \mathrm{d} x, \\
& \int_{0}^{T} \int_{0}^{l}\left\{-\theta(x, t) n_{t}(x, t)-r q^{\varepsilon}(x, t) n_{x}^{\varepsilon}(x, t)+m \psi_{x} n_{t}(x, t)\right\} \mathrm{d} x \mathrm{~d} t \\
= & \int_{0}^{l}\left[\theta(x, 0)-m \psi_{x}(x, 0)\right] n(x, 0) \mathrm{d} x, \\
& \int_{0}^{T} \int_{0}^{l}\left\{-\tau q(x, t) y_{t}(x, t)+q^{\varepsilon}(x, t) y(x, t)+r \theta_{x}(x, t) y(x, t)\right\} \mathrm{d} x \mathrm{~d} t \\
= & \int_{0}^{l} \tau q(x, 0) y(x, 0) \mathrm{d} x,
\end{aligned}
$$

for every $\mathcal{X} \in W^{1,1}\left(0, T ; L^{2}(0, l)\right) \cap L^{2}(0, T ; \mathcal{V})$ such that $\mathcal{X}(\cdot, T)=\psi(\cdot, T)$, for every $n \in$ $W^{1,1}\left(0, T ; L^{2}(0, l) \cap L^{2}(0, T ; \mathcal{H})\right)$ and $y \in W^{1,1}\left(0, T ; L^{2}(0, l) \cap L^{2}(0, T ; \mathcal{V})\right)$ such that $y(\cdot, T)=0$, $n(\cdot, T)=0$.

Here are the main results of the paper.

Theorem 2.2 (Global existence) Under assumption (2.1), there exists a weak solution (in the sense of Definition 2.1) of problem (1.1)-(1.6).

By a regularization, a priori estimates, and passage to the limit procedure, the proof of this result will be carried out in Section 3. In Section 4, we shall prove the following exponential decay result.

Theorem 2.3 (Exponential decay) Let $\varphi$ be a weak solution to problem (1.1)-(1.6) provided by Theorem 2.2. Then there exist two positive constants $R$ and $\omega$, independent of $t$, such that

$$
E(t) \leq R E(0) e^{-\omega t}, \quad \text { for all } t \geq 0
$$




\section{Global existence}

In this section, we show that the solution for problem (1.1)-(1.6) is global. Firstly, in Section 3.1, we approximate problem (1.1)-(1.6) by a penalization procedure and we prove well-posedness for the regularized problem (Proposition 3.1 below). Then, in Section 3.2, we show that a sequence of approximate solutions converges to a solution to the original problem.

\subsection{Approximating problem}

For any $\varepsilon>0$, we introduce the families of initial data $\left(\varphi_{0}^{\varepsilon}, \psi_{0}^{\varepsilon}, \theta_{0}^{\varepsilon}, q_{0}^{\varepsilon}\right)_{\varepsilon>0}$, satisfying

$$
\left(\varphi_{0}^{\varepsilon}, \psi_{0}^{\varepsilon}, \theta_{0}^{\varepsilon}, q_{0}^{\varepsilon}\right) \in\left[H^{2}(0, l) \cap \mathcal{K}\right] \times\left[H^{2}(0, l) \cap \mathcal{V}\right] \times \mathcal{H} \times \mathcal{V},\left(\varphi_{1}^{\varepsilon}, \psi_{1}\right)^{\varepsilon} \in\left[H^{1}(0, l)\right]^{2} .
$$

We introduce a penalized version of problem (1.1)-(1.6) by regularizing the Signorini contact condition with a normal compliance condition. We consider the following system:

$$
\begin{cases}\rho_{1} \varphi_{t t}^{\varepsilon}(x, t)-k\left[\varphi_{x}^{\varepsilon}(x, t)+\psi^{\varepsilon}(x, t)\right]_{x}+\alpha \varphi_{t}^{\varepsilon}(x, t)=0, & (x, t) \in(0, l) \times(0, T), \\ \rho_{2} \psi_{t t}^{\varepsilon}(x, t)-b \psi_{x x}^{\varepsilon}(x, t)+k\left[\varphi_{x}^{\varepsilon}(x, t)+\psi^{\varepsilon}(x, t)\right]-m \theta_{x}^{\varepsilon}(x, t)=0, & (x, t) \in(0, l) \times(0, T), \\ \theta_{t}^{\varepsilon}(x, t)+r q_{x}^{\varepsilon}(x, t)-m \psi_{x t}^{\varepsilon}(x, t)=0, & (x, t) \in(0, l) \times(0, T), \\ \tau q_{t}^{\varepsilon}(x, t)+q^{\varepsilon}(x, t)+r \theta_{x}^{\varepsilon}(x, t)=0, & (x, t) \in(0, l) \times(0, T),\end{cases}
$$

together with

$$
\begin{array}{ll}
\varphi^{\varepsilon}(x, 0)=\varphi_{0}^{\varepsilon}(x), \varphi_{t}^{\varepsilon}(x, 0)=\varphi_{1}^{\varepsilon}(x), & x \in[0, l], \\
\psi^{\varepsilon}(x, 0)=\psi_{0}^{\varepsilon}(x), \psi_{t}^{\varepsilon}(x, 0)=\psi_{1}^{\varepsilon}(x), & x \in[0, l], \\
\theta^{\varepsilon}(x, 0)=\theta_{0}^{\varepsilon}(x), q^{\varepsilon}(x, 0)=q_{0}^{\varepsilon}(x), & x \in[0, l] .
\end{array}
$$

The boundary conditions at $x=0$ are

$$
\varphi^{\varepsilon}(0, t)=0, \psi^{\varepsilon}(0, t)=0, q^{\varepsilon}(0, t)=0, \quad t \in[0, T]
$$

At the tip $x=l$, for $t \in[0, T]$, we set

$$
\psi_{x}^{\varepsilon}(l, t)=0, \theta^{\varepsilon}(l, t)=0, \sigma^{\varepsilon}(t)=\tilde{\sigma}^{\varepsilon}(t),
$$

where

$$
\begin{gathered}
\sigma^{\varepsilon}(t)=k\left[\varphi_{x}^{\varepsilon}(l, t)+\psi^{\varepsilon}(l, t)\right], \\
\tilde{\sigma}^{\varepsilon}(t)=-\frac{1}{\varepsilon}\left\{\left[\varphi^{\varepsilon}(l, t)-g_{1}\right]^{+}-\left[-\varphi^{\varepsilon}(l, t)-g_{2}\right]^{+}\right\}-\varepsilon \varphi_{t}^{\varepsilon}(l, t) .
\end{gathered}
$$

Here and in the sequel, $[f]^{+}:=\max \{f, 0\}$ denotes the positive part of a function $f$.

Henceforth, we will also use the following functionals:

$$
\begin{gathered}
J^{\varepsilon}(t)=\frac{1}{2 \varepsilon}\left\{\left|\left[\varphi^{\varepsilon}(l, t)-g_{1}\right]^{+}\right|^{2}+\left|\left[-\varphi^{\varepsilon}(l, t)-g_{2}\right]^{+}\right|^{2}\right\} \\
\mathcal{E}^{\varepsilon}(t)=E^{\varepsilon}(t)+J^{\varepsilon}(t)
\end{gathered}
$$

where $E^{\varepsilon}(t)=E\left(t, \varphi^{\varepsilon}, \psi^{\varepsilon}, \theta^{\varepsilon}, q^{\varepsilon}\right)$. 
Proposition 3.1 (Existence of an approximate solution) Given any $T>0$, problem (3.2) has a solution

$$
\begin{aligned}
& \varphi^{n, \varepsilon} \in W^{2, \infty}\left(0, T ; L^{2}(0, l)\right) \cap W^{1, \infty}\left(0, T ; H^{1}(0, l)\right) \cap L^{\infty}\left(0, T ; H^{2}(0, l)\right), \\
& \psi^{n, \varepsilon} \in W^{2, \infty}\left(0, T ; L^{2}(0, l)\right) \cap W^{1, \infty}\left(0, T ; H^{1}(0, l)\right) \cap L^{\infty}\left(0, T ; H^{2}(0, l)\right), \\
& \theta^{n, \varepsilon} \in W^{1, \infty}\left(0, T ; L^{2}(0, l)\right) \cap L^{\infty}\left(0, T ; H^{1}(0, l)\right), \\
& q^{n, \varepsilon} \in W^{1, \infty}\left(0, T ; L^{2}(0, l)\right) \cap L^{\infty}\left(0, T ; H^{1}(0, l)\right),
\end{aligned}
$$

with initial data satisfying (3.1), (3.3) and compatible with the boundary conditions (3.4)-(3.6) for $t=0$.

Proof. (Construction of Faedo-Galerkin approximations) Let $\left\{w_{j}\right\}_{j=1}^{\infty}$, be a basis of $\mathcal{V}$ and $\left\{\xi_{j}\right\}_{j=1}^{\infty}$ is basis of $\mathcal{H}$ such that $w_{1}=\varphi_{0}^{\varepsilon}, w_{2}=\varphi_{1}^{\varepsilon}, w_{3}=\psi_{0}^{\varepsilon}, w_{4}=\psi_{1}^{\varepsilon}, w_{5}=q_{0}^{\varepsilon}$ and $\xi_{1}=\theta_{0}^{\varepsilon}$. We construct the approximate solutions of the form

$$
\begin{aligned}
\varphi^{n, \varepsilon}(x, t) & =\sum_{j=1}^{n} h_{j}^{n}(t) w_{j}(x), \psi^{n, \varepsilon}(x, t)=\sum_{j=1}^{n} p_{j}^{n}(t) w_{j}(x), \\
\theta^{n, \varepsilon}(x, t) & =\sum_{j=1}^{n} u_{j}^{n}(t) \xi_{j}(x), q^{n, \varepsilon}(x, t)=\sum_{j=1}^{n} v_{j}^{n}(t) w_{j}(x),
\end{aligned}
$$

verifying, for $j=1, \ldots, n$,

$$
\begin{gathered}
\int_{0}^{l}\left\{\rho_{1} \varphi_{t t}^{n, \varepsilon}(x, t) w_{j}(x)+k\left[\varphi_{x}^{n, \varepsilon}(x, t)+\psi^{n, \varepsilon}(x, t)\right] w_{j x}(x)+\alpha \varphi_{t}^{n, \varepsilon}(x, t) w_{j}(x)\right\} \mathrm{d} x \\
-\tilde{\sigma}^{n, \varepsilon}(t) w_{j}(l)=0, \\
\int_{0}^{l}\left\{\rho_{2} \psi_{t t}^{n, \varepsilon}(x, t) w_{j}(x)+b \psi_{x}^{n, \varepsilon}(x, t) w_{j x}(x)+k\left[\varphi_{x}^{n, \varepsilon}(x, t)+\psi^{n, \varepsilon}(x, t)\right] w_{j}(x)\right. \\
\left.-m \theta_{x}^{n, \varepsilon}(x, t) w_{j}(x)\right\} \mathrm{d} x=0, \\
\int_{0}^{l}\left[\theta_{t}^{n, \varepsilon}(x, t) \xi_{j}(x)-r q^{n, \varepsilon}(x, t) \xi_{j x}(x)+m \psi_{t}^{n, \varepsilon}(x, t) \xi_{j x}(x)\right] \mathrm{d} x=0, \\
\int_{0}^{l}\left[\tau q_{t}^{n, \varepsilon}(x, t) w_{j}(x)+q^{n, \varepsilon}(x, t) w_{j}(x)-r \theta^{n, \varepsilon}(x, t) w_{j x}(x)\right] \mathrm{d} x=0,
\end{gathered}
$$

where

$$
\tilde{\sigma}^{n, \varepsilon}(t)=-\frac{1}{\varepsilon}\left\{\left[\varphi^{n, \varepsilon}(l, t)-g_{1}\right]^{+}-\left[-\varphi^{n, \varepsilon}(l, t)-g_{2}\right]^{+}\right\}-\varepsilon \varphi_{t}^{n, \varepsilon}(l, t)
$$

and initial data

$$
\begin{aligned}
& \varphi^{n, \varepsilon}(x, 0)=\varphi_{0}^{\varepsilon}(x), \quad \varphi_{t}^{n, \varepsilon}(x, 0)=\varphi_{1}^{\varepsilon}(x), \quad x \in[0, l], \\
& \psi^{n, \varepsilon}(x, 0)=\psi_{0}^{\varepsilon}(x), \quad \psi_{t}^{n, \varepsilon}(x, 0)=\psi_{1}^{\varepsilon}(x), \quad x \in[0, l], \\
& \theta^{n, \varepsilon}(x, 0)=\theta_{0}^{\varepsilon}(x), \quad q^{n, \varepsilon}(x, 0)=q_{0}^{\varepsilon}(x), \quad x \in[0, l] .
\end{aligned}
$$

Accordingly, the standard theory of ordinary differential equations guarantees, under Lipschitz conditions, system (3.10)-(3.13) appended by initial conditions (3.14) admits a local solution. We 
now need the a priori estimates that permit us to extend the solution to the whole interval $[0, T]$, for any $T>0$.

(A priori estimates) We multiply (3.10) by $h_{j t}^{n},(3.11)$ by $p_{j t}^{n},(3.12)$ by $u_{j}^{n},(3.13)$ by $v_{j}^{n}$, respectively, summing over $j$ and adding the resulting equations, we infer

$$
\frac{\mathrm{d}}{\mathrm{d} t} E^{n, \varepsilon}(t)+\alpha \int_{0}^{l}\left|\varphi_{t}^{n, \varepsilon}(x, t)\right|^{2} \mathrm{~d} x+\int_{0}^{l}\left|q^{n, \varepsilon}(x, t)\right|^{2} \mathrm{~d} x=\tilde{\sigma}^{n, \varepsilon}(t) \varphi_{t}^{n, \varepsilon}(l, t),
$$

where $E^{n, \varepsilon}(t)=E\left(t, \varphi^{n, \varepsilon}, \psi^{n, \varepsilon}, \theta^{n, \varepsilon}, q^{n, \varepsilon}\right)$. Note that if we denote by $f^{-}=\max \{-f, 0\}$ the negative part of a function $f$, we have $f^{+} f_{t}=f^{+}\left(f^{+}-f^{-}\right)_{t}=f^{+} f_{t}^{+}=\frac{1}{2} \frac{\mathrm{d}}{\mathrm{d} t}\left[f^{+}\right]^{2}$. Thus, the previous equality becomes

$$
\frac{\mathrm{d}}{\mathrm{d} t} \mathcal{E}^{n, \varepsilon}(t)+\alpha \int_{0}^{l}\left|\varphi_{t}^{n, \varepsilon}(x, t)\right|^{2} \mathrm{~d} x+\int_{0}^{l}\left|q^{n, \varepsilon}(x, t)\right|^{2} \mathrm{~d} x+\varepsilon\left|\varphi_{t}^{n, \varepsilon}(l, t)\right|^{2}=0 .
$$

An integration over $(0, t)$ and initial conditions (3.14) ensure that

$$
E^{n, \varepsilon}(t)+\frac{1}{2 \varepsilon}\left\{\left|\left[\varphi^{n, \varepsilon}(l, t)-g_{1}\right]^{+}\right|^{2}+\left|\left[-\varphi^{n, \varepsilon}(l, t)-g_{2}\right]^{+}\right|^{2}\right\} \leq K,
$$

where $K$ is a positive constant independent of $n$. Note that for any $\varphi_{0}^{\varepsilon} \in \mathcal{K}$ we get that $\tilde{\sigma}^{n, \varepsilon}(0)=$ $-\varepsilon \varphi_{t}^{n, \varepsilon}(l, 0)$.

After a differentiation of Eqs. (3.10)-(3.13) with respect to t, we have

$$
\begin{gathered}
\int_{0}^{l}\left\{\rho_{1} \varphi_{t t t}^{n, \varepsilon}(x, t) w_{j}(x)+k\left[\varphi_{x t}^{n, \varepsilon}(x, t)+\psi_{t}^{n, \varepsilon}(x, t)\right] w_{j x}(x)+\alpha \varphi_{t t}^{n, \varepsilon}(x, t) w_{j}(x)\right\} \mathrm{d} x \\
-\tilde{\sigma}_{t}^{n, \varepsilon}(t) w_{j}(l)=0 \\
\int_{0}^{l}\left\{\rho_{2} \psi_{t t t}^{n, \varepsilon}(x, t) w_{j}(x)+b \psi_{x t}^{n, \varepsilon}(x, t) w_{j x}(x)+k\left[\varphi_{x t}^{n, \varepsilon}(x, t)+\psi_{t}^{n, \varepsilon}(x, t)\right] w_{j}(x)\right. \\
\left.-m \theta_{x t}^{n, \varepsilon}(x, t) w_{j}(x)\right\} \mathrm{d} x=0, \\
\int_{0}^{l}\left[\theta_{t t}^{n, \varepsilon}(x, t) \xi_{j}(x)-r q_{t}^{n, \varepsilon}(x, t) \xi_{j x}(x)+m \psi_{t t}^{n, \varepsilon}(x, t) \xi_{j x}(x)\right] \mathrm{d} x=0, \\
\int_{0}^{l}\left[\tau q_{t t}^{n, \varepsilon}(x, t) w_{j}(x)+q_{t}^{n, \varepsilon}(x, t) w_{j}(x)-r \theta_{t}^{n, \varepsilon}(x, t) w_{j x}(x)\right] \mathrm{d} x=0 .
\end{gathered}
$$

We multiply (3.16) by $h_{j t t}^{n}$, (3.17) by $p_{j t t}^{n}$, (3.18) by $u_{j t}^{n}$, (3.19) by $v_{j t}^{n}$, summing over $j$ and adding the resulting equations, we have

$$
\frac{\mathrm{d}}{\mathrm{d} t} E_{t}^{n, \varepsilon}(t)+\alpha \int_{0}^{l}\left|\varphi_{t t}^{n, \varepsilon}(x, t)\right|^{2} \mathrm{~d} x+\int_{0}^{l}\left|q_{t}^{n, \varepsilon}(x, t)\right|^{2} \mathrm{~d} x+\varepsilon\left|\varphi_{t t}^{n, \varepsilon}(l, t)\right|^{2}=-\frac{1}{\varepsilon} B^{n}(t) \varphi_{t t}^{n, \varepsilon}(l, t),
$$

where $E_{t}^{n, \varepsilon}(t)=E\left(t, \varphi_{t}^{n, \varepsilon}, \psi_{t}^{n, \varepsilon}, \theta_{t}^{n, \varepsilon}, q_{t}^{n, \varepsilon}\right)$ and $E_{t}^{n, \varepsilon}(t), B^{n}(t)$ are defined as follows

$$
E_{t}^{n, \varepsilon}(t):=\frac{1}{2} \int_{0}^{l}\left[\rho_{1}\left|\varphi_{t t}^{n, \varepsilon}(x, t)\right|^{2}+\rho_{2}\left|\psi_{t t}^{n, \varepsilon}(x, t)\right|^{2}+b\left|\psi_{x t}(x, t)\right|^{2}\right] \mathrm{d} x
$$




$$
\begin{aligned}
& +\frac{1}{2} \int_{0}^{l}\left[k\left|\varphi_{x t}^{n, \varepsilon}(x, t)+\psi_{t}^{n, \varepsilon}(x, t)\right|^{2}+\tau\left|q_{t}^{n, \varepsilon}(x, t)\right|^{2}+\left|\theta_{t}^{n, \varepsilon}(x, t)\right|^{2}\right] \mathrm{d} x, \\
B^{n}(t)= & \frac{\mathrm{d}}{\mathrm{d} t}\left\{\left[\varphi^{n, \varepsilon}(l, t)-g_{1}\right]^{+}-\left[-\varphi^{n, \varepsilon}(l, t)-g_{2}\right]^{+}\right\} .
\end{aligned}
$$

In addition, by applying the Young's and Sobolev's inequalities and noting that $\left|\left(f^{+}\right)_{t}\right| \leq\left|f_{t}\right|$, we can estimate the last term in (3.20) as follows (see [7])

$$
\begin{aligned}
& \frac{1}{\varepsilon}\left|B^{n}(t)\right|\left|\varphi_{t t}^{n, \varepsilon}(l, t)\right| \\
\leq & \frac{\varepsilon}{2}\left|\varphi_{t t}^{n, \varepsilon}(l, t)\right|^{2}+C_{\varepsilon}\left|B^{n}(t)\right|^{2} \\
\leq & \frac{\varepsilon}{2}\left|\varphi_{t t}^{n, \varepsilon}(l, t)\right|^{2}+C_{\varepsilon} \int_{0}^{l}\left|\varphi_{x t}^{n, \varepsilon}(x, t)+\psi_{t}^{n, \varepsilon}(x, t)\right|^{2} \mathrm{~d} x+C_{\varepsilon} \int_{0}^{l}\left|\psi_{x t}^{n, \varepsilon}(x, t)\right|^{2} \mathrm{~d} x,
\end{aligned}
$$

where $C_{\varepsilon}$ is a positive constant depending on $\varepsilon$ but independent of $n$, which is allowed to vary even in the same formula. From (3.20), we have

$$
\begin{aligned}
& \frac{\mathrm{d}}{\mathrm{d} t} E_{t}^{n, \varepsilon}(t)+\alpha \int_{0}^{l}\left|\varphi_{t t}^{n, \varepsilon}(x, t)\right|^{2} \mathrm{~d} x+\int_{0}^{l}\left|q_{t}^{n, \varepsilon}(x, t)\right|^{2} \mathrm{~d} x+\frac{\varepsilon}{2}\left|\varphi_{t t}^{n, \varepsilon}(l, t)\right|^{2} \\
\leq & C_{\varepsilon} \int_{0}^{l}\left|\varphi_{x t}^{n, \varepsilon}(x, t)+\psi_{t}^{n, \varepsilon}(x, t)\right|^{2} \mathrm{~d} x+C_{\varepsilon} \int_{0}^{l}\left|\psi_{x t}^{n, \varepsilon}(x, t)\right|^{2} \mathrm{~d} x .
\end{aligned}
$$

An integration over $(0, t)$ implies

$$
\begin{aligned}
& E_{t}^{n, \varepsilon}(t)+\int_{0}^{t} \int_{0}^{l}\left[\alpha\left|\varphi_{t t}^{n, \varepsilon}(x, t)\right|^{2}+\left|q_{t}^{n, \varepsilon}(x, t)\right|^{2}\right] \mathrm{d} x \mathrm{~d} t \\
\leq & E_{t}^{n, \varepsilon}(0)+C_{\varepsilon} \int_{0}^{t} \int_{0}^{l}\left[\left|\varphi_{x t}^{n, \varepsilon}(x, t)+\psi_{t}^{n, \varepsilon}(x, t)\right|^{2}+\left|\psi_{x t}^{n, \varepsilon}(x, t)\right|^{2}\right] \mathrm{d} x \mathrm{~d} t .
\end{aligned}
$$

We can show that the second order energy is initially bounded, independently of $n$, namely

$$
\begin{aligned}
E_{t}^{n, \varepsilon}(0):= & \frac{1}{2} \int_{0}^{l}\left[\rho_{1}\left|\varphi_{t t}^{n, \varepsilon}(x, 0)\right|^{2}+\rho_{2}\left|\psi_{t t}^{n, \varepsilon}(x, 0)\right|^{2}+\left|\theta_{t}^{n, \varepsilon}(x, 0)\right|^{2}+\tau\left|q_{t}^{n, \varepsilon}(x, 0)\right|^{2}\right] \mathrm{d} x \\
& +\frac{1}{2} \int_{0}^{l}\left[k\left|\varphi_{1 x}^{n, \varepsilon}(x)+\psi_{1}^{n, \varepsilon}(x)\right|^{2}+b\left|\psi_{1 x}^{n, \varepsilon}(x)\right|^{2}\right] \mathrm{d} x
\end{aligned}
$$

is bounded independently of $n$. To this aim, we multiply (3.10) by $h_{j t t}^{n}$, we sum up over $j=1, \ldots, n$ and we let $t \rightarrow 0$. By (3.14), we have

$$
\begin{aligned}
& \int_{0}^{l}\left\{\rho_{1}\left|\varphi_{t t}^{n, \varepsilon}(x, 0)\right|^{2}+k\left[\varphi_{0 x}^{\varepsilon}(x)+\psi_{0}^{\varepsilon}(x)\right] \varphi_{x t t}^{n, \varepsilon}(x, 0)+\alpha \varphi_{1}^{\varepsilon}(x) \varphi_{t t}^{n, \varepsilon}(x, 0)\right\} \mathrm{d} x \\
& -\tilde{\sigma}^{n, \varepsilon}(0) \varphi_{t t}^{n, \varepsilon}(l, 0)=0 .
\end{aligned}
$$

After an integration by parts and owing to the compatibility conditions (3.4)-(3.6) for $t=0$, we find

$$
\begin{aligned}
& \int_{0}^{l}\left\{\rho_{1}\left|\varphi_{t t}^{n, \varepsilon}(x, 0)\right|^{2}-k\left[\varphi_{0 x x}^{\varepsilon}(x)+\psi_{0 x}^{\varepsilon}(x)\right] \varphi_{t t}^{n, \varepsilon}(x, 0)+\alpha \varphi_{1}^{\varepsilon}(x) \varphi_{t t}^{n, \varepsilon}(x, 0)\right\} \mathrm{d} x \\
& +\left\{k\left[\varphi_{0 x}^{\varepsilon}(l)+\psi_{0}^{\varepsilon}(l)\right]-\tilde{\sigma}^{n, \varepsilon}(0)\right\} \varphi_{t t}^{n, \varepsilon}(l, 0)=0
\end{aligned}
$$


In the light of Hölder's inequality and Young's inequality, we deduce that there exists a constant $C$ independent of $n$ such that

$$
\int_{0}^{l}\left|\varphi_{t t}^{n, \varepsilon}(x, 0)\right|^{2} \mathrm{~d} x \leq C \int_{0}^{l}\left[\left|\varphi_{0 x x}^{\varepsilon}(x)\right|^{2}+\left|\psi_{0 x}^{\varepsilon}(x)\right|^{2}+\left|\varphi_{1}^{\varepsilon}(x)\right|^{2}\right] \mathrm{d} x \leq C .
$$

Similarly, multiplying (3.11) by $p_{j t t}^{n},(3.12)$ by $u_{j t}^{n}$, summing up over $j=1, \ldots, n$ and letting $t \rightarrow 0$, we get

$$
\int_{0}^{l}\left|\psi_{t t}^{n, \varepsilon}(x, 0)\right|^{2} \mathrm{~d} x \leq C \int_{0}^{l}\left[\left|\psi_{0 x x}^{\varepsilon}(x)\right|^{2}+\left|\varphi_{0 x}^{\varepsilon}(x)\right|^{2}+\left|\theta_{0 x}^{\varepsilon}(x)\right|^{2}\right] \mathrm{d} x \leq C
$$

and

$$
\int_{0}^{l}\left\{\left|\theta_{t}^{n, \varepsilon}(x, 0)\right|^{2}+r q_{0 x}^{\varepsilon}(x) \theta_{t}^{n, \varepsilon}(x, 0)-m \psi_{1 x}^{\varepsilon}(x) \theta_{t}^{n, \varepsilon}(x, 0)\right\} \mathrm{d} x=0,
$$

which leads to the inequality

$$
\int_{0}^{l}\left|\theta_{t}^{n, \varepsilon}(x, 0)\right|^{2} \mathrm{~d} x \leq C \int_{0}^{l}\left[\left|q_{0 x}^{\varepsilon}(x)\right|^{2}+\left|\psi_{1 x}^{\varepsilon}(x)\right|^{2}\right] \mathrm{d} x \leq C .
$$

Finally, multiplying (3.13) by $v_{j t}^{n}$, summing up over $j=1, \ldots, n$ and letting $t \rightarrow 0$, we obtain

$$
\int_{0}^{l}\left\{\tau\left|q_{t}^{n, \varepsilon}(x, 0)\right|^{2}+q_{0}^{n, \varepsilon}(x) q_{t}^{n, \varepsilon}(x)-r \theta_{0 x}^{n, \varepsilon} q_{t}^{n, \varepsilon}(x, 0)\right\} \mathrm{d} x=0,
$$

then, we have

$$
\int_{0}^{l}\left|q_{t}^{n, \varepsilon}(x, 0)\right|^{2} \mathrm{~d} x \leq C \int_{0}^{l}\left[\left|q_{0}^{\varepsilon}(x)\right|^{2}+\left|\theta_{0 x}^{\varepsilon}(x)\right|^{2}\right] \mathrm{d} x \leq C .
$$

By (3.1), we infer that

$$
E_{t}^{n, \varepsilon}(0) \leq C \int_{0}^{l}\left[\left|\varphi_{0 x x}^{\varepsilon}(x)\right|^{2}+\left|\psi_{0 x x}^{\varepsilon}(x)\right|^{2}+\left|\theta_{0 x}^{\varepsilon}(x)\right|^{2}+\left|q_{0}^{\varepsilon}(x)\right|^{2}+\left|\varphi_{1}^{\varepsilon}(x)\right|^{2}+\left|\psi_{1 x}^{\varepsilon}(x)\right|^{2}\right] \mathrm{d} x \leq C .
$$

Thus, from (3.22) and applying Gronwall's inequality, we find that $E_{t}^{n, \varepsilon}(t)$ is bounded in $[0, T]$. (Passage to the limit) Inequalities (3.15) and (3.22) guarantee that

$$
\begin{array}{r}
\varphi^{n, \varepsilon} \text { is bounded in } W^{2, \infty}\left(0, T ; L^{2}(0, l)\right) \cap W^{1, \infty}\left(0, T ; H^{1}(0, l)\right), \\
\psi^{n, \varepsilon} \text { is bounded in } W^{2, \infty}\left(0, T ; L^{2}(0, l)\right) \cap W^{1, \infty}\left(0, T ; H^{1}(0, l)\right), \\
\theta^{n, \varepsilon} \text { is bounded in } W^{1, \infty}\left(0, T ; L^{2}(0, l)\right), \\
q^{n, \varepsilon} \text { is bounded in } W^{1, \infty}\left(0, T ; L^{2}(0, l)\right), \\
{\left[\varphi^{n, \varepsilon}(l, t)-g_{1}\right]^{+} \text {is bounded in } L^{\infty}(0, T),} \\
{\left[-\varphi^{n, \varepsilon}(l, t)-g_{2}\right]^{+} \text {is bounded in } L^{\infty}(0, T) .}
\end{array}
$$

Therefore we deduce, up to a subsequence, the convergence

$$
\varphi^{n, \varepsilon} \rightarrow \varphi^{\varepsilon} \quad \text { weak }^{*} \text { in } W^{2, \infty}\left(0, T ; L^{2}(0, l)\right) \cap W^{1, \infty}\left(0, T ; H^{1}(0, l)\right),
$$




$$
\begin{array}{r}
\psi^{n, \varepsilon} \rightarrow \psi^{\varepsilon} \quad \text { weak }^{*} \text { in } W^{2, \infty}\left(0, T ; L^{2}(0, l)\right) \cap W^{1, \infty}\left(0, T ; H^{1}(0, l)\right), \\
\theta^{n, \varepsilon} \rightarrow \theta^{\varepsilon} \quad \text { weak }^{*} \text { in } W^{1, \infty}\left(0, T ; L^{2}(0, l)\right), \\
q^{n, \varepsilon} \rightarrow q^{\varepsilon} \quad \text { weak }^{*} \text { in } W^{1, \infty}\left(0, T ; L^{2}(0, l)\right), \\
{\left[\varphi^{n, \varepsilon}(l, t)-g_{1}\right]^{+} \rightarrow\left[\varphi^{\varepsilon}(l, t)-g_{1}\right]^{+} \quad \text { weak }^{*} \text { in } L^{\infty}(0, T),} \\
{\left[-\varphi^{n, \varepsilon}(l, t)-g_{2}\right]^{+} \rightarrow\left[-\varphi^{n, \varepsilon}(l, t)-g_{2}\right]^{+} \quad \text { weak }^{*} \text { in } L^{\infty}(0, T) .}
\end{array}
$$

By standard procedure, by letting $n \rightarrow \infty$ in (3.10), we recover (3.2) and the initial and boundary conditions (3.3)-(3.6). In particular, from equations $(3.2)_{3}$ and $(3.2)_{4}$, we deduce that $\theta_{x}^{\varepsilon}, q_{x}^{\varepsilon} \in$ $L^{\infty}\left(0, T ; L^{2}(0, l)\right)$ and hence the regularity $\left(\varphi^{\varepsilon}, \psi^{\varepsilon}, \theta^{\varepsilon}, q^{\varepsilon}\right)$ verifies the regularity specified in (3.9).

Proposition 3.2 (Uniqueness) For any $T>0$, the solution $\left(\varphi^{\varepsilon}, \psi^{\varepsilon}, \theta^{\varepsilon}, q^{\varepsilon}\right)$ to problem (3.2), with initial data satisfying (3.3) and compatible with the boundary conditions (3.4)-(3.6), is unique.

Proof. Let $\left(\varphi^{\varepsilon}, \psi^{\varepsilon}, \theta^{\varepsilon}, q^{\varepsilon}\right)$ and $\left(\Phi^{\varepsilon}, \Psi^{\varepsilon}, \Theta^{\varepsilon}, \Upsilon^{\varepsilon}\right)$ be two solutions of (3.2), (3.4)-(3.6) whose regularity is specified by (3.9). We define

$$
U^{\varepsilon}:=\varphi^{\varepsilon}-\Phi^{\varepsilon}, Q^{\varepsilon}: \psi^{\varepsilon}-\Psi^{\varepsilon}, R^{\varepsilon}:=\theta^{\varepsilon}-\Theta^{\varepsilon}, S^{\varepsilon}:=q^{\varepsilon}-\Upsilon^{\varepsilon},
$$

satisfying

$$
\begin{cases}\rho_{1} U_{t t}^{\varepsilon}(x, t)-k\left[U_{x}^{\varepsilon}(x, t)+Q^{\varepsilon}(x, t)\right]_{x}+\alpha U_{t}^{\varepsilon}(x, t)=0, & (x, t) \in(0, l) \times(0, T), \\ \rho_{2} Q_{t t}^{\varepsilon}(x, t)-b Q_{x x}^{\varepsilon}(x, t)+k\left[U_{x}^{\varepsilon}(x, t)+Q^{\varepsilon}(x, t)\right]-m R_{x}^{\varepsilon}(x, t)=0, & (x, t) \in(0, l) \times(0, T), \\ R_{t}^{\varepsilon}(x, t)+r S_{x}^{\varepsilon}(x, t)-m Q_{x t}^{\varepsilon}(x, t)=0, & (x, t) \in(0, l) \times(0, T), \\ \tau S_{t}^{\varepsilon}(x, t)+S^{\varepsilon}(x, t)+r R_{x}^{\varepsilon}(x, t)=0, & (x, t) \in(0, l) \times(0, T),\end{cases}
$$

with the initial conditions

$$
\begin{array}{lll}
U^{\varepsilon}(x, 0)=0, & U_{t}^{\varepsilon}(x, 0)=0, & x \in[0, l], \\
Q^{\varepsilon}(x, 0)=0, & Q_{t}^{\varepsilon}(x, 0)=0, & x \in[0, l], \\
R^{\varepsilon}(x, 0)=0, & S^{\varepsilon}(x, 0)=0, & x \in[0, l]
\end{array}
$$

and

$$
\begin{gathered}
U^{\varepsilon}(0, t)=0, \quad Q_{t}^{\varepsilon}(0, t)=0, \quad R^{\varepsilon}(0, t)=0, \quad S^{\varepsilon}(0, t)=0, \\
Q^{\varepsilon}(l, t)=Q_{x}^{\varepsilon}(l, t)=0, \quad R^{\varepsilon}(l, t)=0, \quad S^{\varepsilon}(l, t)=0, \quad \varsigma^{\varepsilon}(t)=\tilde{\varsigma}^{\varepsilon}(t), \quad t \in[0, T],
\end{gathered}
$$

where

$$
\begin{gathered}
\varsigma^{\varepsilon}(t)=k\left[U_{x}^{\varepsilon}(l, t)+Q^{\varepsilon}(l, t)\right], \\
\tilde{\varsigma}^{\varepsilon}(t)=-\frac{1}{\varepsilon}\left\{\left[\varphi^{\varepsilon}(l, t)-g_{1}\right]^{+}-\left[-\varphi^{\varepsilon}(l, t)-g_{2}\right]^{+}-\left[\Phi^{\varepsilon}(l, t)-g_{1}\right]^{+}+\left[-\Phi^{\varepsilon}(l, t)-g_{2}\right]^{+}\right\}-\varepsilon U_{t}^{\varepsilon}(l, t) .
\end{gathered}
$$

Multiplying $(3.16)$ by $U_{t}^{\varepsilon},(3.17)$ by $Q_{t}^{\varepsilon},(3.18)$ by $R^{\varepsilon},(3.19)$ by $S^{\varepsilon}$ and integrating over $[0, l]$, we get

$$
\frac{\mathrm{d}}{\mathrm{d} t} E\left(t,\left(U^{\varepsilon}, Q^{\varepsilon}, R^{\varepsilon}, S^{\varepsilon}\right)\right)+\alpha \int_{0}^{l}\left|U_{t}^{\varepsilon}(x, t)\right|^{2} \mathrm{~d} x+\int_{0}^{l}\left|S^{\varepsilon}(x, t)\right|^{2} \mathrm{~d} x=\tilde{\varsigma}^{\varepsilon}(t) U_{t}^{\varepsilon}(l, t) .
$$


In view of the relation $\left|f^{+}-g^{+}\right| \leq|f-g|$, we obtain the estimate

$$
\begin{aligned}
& \left|\left[\varphi^{\varepsilon}(l, t)-g_{1}\right]^{+}-\left[-\varphi^{\varepsilon}(l, t)-g_{2}\right]^{+}+\left[\Phi^{\varepsilon}(l, t)-g_{1}\right]^{+}-\left[-\Phi^{\varepsilon}(l, t)-g_{2}\right]^{+}\right| \\
\leq & 2\left|\varphi^{\varepsilon}(l, t)-\Phi^{\varepsilon}(l, t)\right|=2\left|U^{\varepsilon}(l, t)\right| .
\end{aligned}
$$

By means of Poincaré's inequality and the Sobolev embedding theorem, we have

$$
\begin{aligned}
\tilde{\varsigma}^{\varepsilon}(t) U_{t}^{\varepsilon}(l, t)= & -\frac{1}{\varepsilon}\left\{\left[\varphi^{\varepsilon}(l, t)-g_{1}\right]^{+}-\left[-\varphi^{\varepsilon}(l, t)-g_{2}\right]^{+}-\left[\Phi^{\varepsilon}(l, t)-g_{1}\right]^{+}\right. \\
& \left.+\left[-\Phi^{\varepsilon}(l, t)-g_{2}\right]^{+}\right\} U_{t}^{\varepsilon}(l, t)-\varepsilon\left|U_{t}^{\varepsilon}(l, t)\right|^{2} \\
\leq & \frac{2}{\varepsilon}\left|U^{\varepsilon}(l, t)\right|\left|U_{t}^{\varepsilon}(l, t)\right|-\varepsilon\left|U_{t}^{\varepsilon}(l, t)\right|^{2} \\
\leq & -\frac{\varepsilon}{2}\left|U_{t}^{\varepsilon}(l, t)\right|^{2}+C_{\varepsilon}\left|U^{\varepsilon}(l, t)\right|^{2} \\
\leq & -\frac{\varepsilon}{2}\left|U_{t}^{\varepsilon}(l, t)\right|^{2}+C_{\varepsilon} \int_{0}^{l}\left|U_{x}^{\varepsilon}(x, t)\right|^{2} \mathrm{~d} x
\end{aligned}
$$

A substitution into (3.26) leads to

$$
\frac{\mathrm{d}}{\mathrm{d} t} E\left(t, U^{\varepsilon}, Q^{\varepsilon}, R^{\varepsilon}, S^{\varepsilon}\right)+\alpha \int_{0}^{l}\left|U_{t}^{\varepsilon}(x, t)\right|^{2} \mathrm{~d} x+\int_{0}^{l}\left|S^{\varepsilon}(x, t)\right|^{2} \mathrm{~d} x+\frac{\varepsilon}{2}\left|U_{t}^{\varepsilon}(l, t)\right|^{2} \leq C E^{\varepsilon}(t) .
$$

In view of initial conditions (3.23)-(3.25), $E\left(0, U^{\varepsilon}, Q^{\varepsilon}, R^{\varepsilon}, S^{\varepsilon}\right)=0$. Thus, by the Gronwall lemma, we find that $E\left(t, U^{\varepsilon}, Q^{\varepsilon}, R^{\varepsilon}, S^{\varepsilon}\right)=0$ on $[0, T]$. This implies that $\left(\varphi^{\varepsilon}, \psi^{\varepsilon}, \theta^{\varepsilon}, q^{\varepsilon}\right)=$ $\left(\Phi^{\varepsilon}, \Psi^{\varepsilon}, \Theta^{\varepsilon}, \Upsilon^{\varepsilon}\right)$, and our conclusion follows.

\subsection{Proof of Theorem 2.2}

The idea is to consider a sequence of approximate solutions (provided by Proposition 3.1) and to show their convergence (as $\varepsilon \rightarrow 0$ ) to a weak solution of problem (1.1)-(1.6). Given data $\left(\varphi_{0}^{\varepsilon}, \psi_{0}^{\varepsilon}, \theta_{0}^{\varepsilon}, q_{0}^{\varepsilon}\right) \in \mathcal{K} \times \mathcal{V} \times L^{2}(0, l) \times L^{2}(0, l),\left(\varphi_{1}, \psi_{1}\right) \in\left[L^{2}(0, l)\right]^{2}$, let us consider the sequences of functions $\left(\varphi_{0}^{\varepsilon}, \psi_{0}^{\varepsilon}, \theta_{0}^{\varepsilon}, q_{0}^{\varepsilon}\right),\left(\varphi_{1}^{\varepsilon}, \psi_{1}^{\varepsilon}\right)$ with the regularity expressed in (3.1) and such that

$$
\begin{aligned}
& \left(\varphi_{0}^{\varepsilon}, \psi_{0}^{\varepsilon}, \theta_{0}^{\varepsilon}, q_{0}^{\varepsilon}\right) \rightarrow\left(\varphi_{0}, \psi_{0}, \theta_{0}, q_{0}\right) \in \mathcal{V} \times \mathcal{V} \times L^{2}(0, l) \times L^{2}(0, l), \\
& \left(\varphi_{1}^{\varepsilon}, \psi_{1}^{\varepsilon}\right) \rightarrow\left(\varphi_{1}, \psi_{1}\right) \in\left[L^{2}(0, l)\right]^{2} .
\end{aligned}
$$

Multiplying equations $(3.2)_{1},(3.2)_{2},(3.2)_{3},(3.2)_{4}$, by $\varphi_{t}^{\varepsilon}, \psi_{t}^{\varepsilon}, \theta^{\varepsilon}, q^{\varepsilon}$ and summing up the resulting equations. An integration over $(0, l)$ and boundary conditions (3.4)-(3.6) lead to

$$
\frac{\mathrm{d}}{\mathrm{d} t} \mathcal{E}^{\varepsilon}(t)+\alpha \int_{0}^{l}\left|\varphi_{t}^{\varepsilon}(x, t)\right|^{2} \mathrm{~d} x+\int_{0}^{l}\left|q^{\varepsilon}(x, t)\right|^{2} \mathrm{~d} x+\varepsilon\left|\varphi_{t}^{\varepsilon}(l, t)\right|^{2}=0,
$$

where the functional $\mathcal{E}^{\varepsilon}$ is defined in (3.8). Now we integrate over $t$, by $(3.1)_{1}$ and $J^{\varepsilon}(0)=0$, we have

$$
\mathcal{E}^{\varepsilon}(t)+\int_{0}^{t}\left[\alpha \int_{0}^{l}\left|\varphi_{t}^{\varepsilon}(x, s)\right|^{2} \mathrm{~d} x+\int_{0}^{l}\left|q^{\varepsilon}(x, s)\right|^{2} \mathrm{~d} x+\varepsilon\left|\varphi_{t}^{\varepsilon}(l, s)\right|^{2}\right] \mathrm{d} s \leq E^{\varepsilon}(0) \leq K,
$$

where $K$ is a positive constant independent of $\varepsilon$. By (3.29), we obtain the estimate

$$
J^{\varepsilon}(t)=\frac{1}{2 \varepsilon}\left\{\left|\left[\varphi^{\varepsilon}(l, t)-g_{1}\right]^{+}\right|^{2}+\left|\left[-\varphi^{\varepsilon}(l, t)-g_{2}\right]^{+}\right|^{2}\right\} \leq K
$$


The boundedness of $E^{\varepsilon}(t)$ implies the existence of a subsequence, we get

$$
\begin{array}{ll}
\varphi^{\varepsilon} \rightarrow \varphi & \text { weak }{ }^{*} \text { in } W^{1, \infty}\left(0, T ; L^{2}(0, l)\right) \cap L^{\infty}\left(0, T ; H^{1}(0, l)\right), \\
\psi^{\varepsilon} \rightarrow \psi & \text { weak* in } W^{1, \infty}\left(0, T ; L^{2}(0, l)\right) \cap L^{\infty}\left(0, T ; H^{1}(0, l)\right), \\
\theta^{\varepsilon} \rightarrow \theta & \text { weak }^{*} \text { in } L^{\infty}\left(0, T ; L^{2}(0, l)\right), \\
q^{\varepsilon} \rightarrow q & \text { weak* in } L^{\infty}\left(0, T ; L^{2}(0, l)\right) .
\end{array}
$$

Moreover, we have

$$
\varepsilon \varphi_{t}^{\varepsilon}(l, \cdot) \rightarrow 0 \quad \text { in } L^{2}(0, T)
$$

Next, we will prove that $(\varphi, \psi, \theta, q)$ is a weak solution to problem (1.1)-(1.6). Inequality (3.30) assures that $\varphi(\cdot, t) \in \mathcal{K}$ for all $t \in[0, T]$. Now, let $\omega \in W^{1,1}\left(0, T ; L^{2}(0, l)\right) \cap L^{2}(0, T ; \mathcal{K})$ such that $\omega(\cdot, T)=\varphi(\cdot, T)$. Multiplying $(3.2)_{1}$ by $\omega-\varphi^{\varepsilon}$ and integrate over $(0, T) \times(0, l)$. By taking (3.5)-(3.6) into account, we obtain

$$
\begin{aligned}
& \int_{0}^{T} \int_{0}^{l}\left\{-\rho_{1} \varphi_{t}^{\varepsilon}(x, t)\left[\omega_{t}(x, t)-\varphi_{t}^{\varepsilon}(x, t)\right]+k\left[\varphi_{x}^{\varepsilon}(x, t)+\psi^{\varepsilon}(x, t)\right]\left[\omega_{x}(x, t)-\varphi_{x}^{\varepsilon}(x, t)\right]\right. \\
& \left.\quad+\alpha \varphi_{t}^{\varepsilon}(x, t)\left[\omega(x, t)-\varphi^{\varepsilon}(x, t)\right]\right\} \mathrm{d} x \mathrm{~d} t \geq \rho_{1} \int_{0}^{l} \varphi_{1}^{\varepsilon}(x)\left[\omega(x, 0)-\varphi_{0}^{\varepsilon}(x)\right] \mathrm{d} x .
\end{aligned}
$$

Similarly, from $(3.2)_{2},(3.2)_{3}$ and $(3.2)_{4}$, we have

$$
\begin{aligned}
\int_{0}^{T} & \int_{0}^{l}\left\{-\rho_{2} \psi_{t}^{\varepsilon}(x, t) \mathcal{X}_{t}(x, t)+b \psi_{x}^{\varepsilon} \mathcal{X}_{x}(x, t)+k\left[\varphi_{x}^{\varepsilon}(x, t)+\psi^{\varepsilon}(x, t)\right] \mathcal{X}(x, t)\right. \\
& \left.+m \theta^{\varepsilon}(x, t) \mathcal{X}_{x}(x, t)\right\} \mathrm{d} x \mathrm{~d} t=\rho_{2} \int_{0}^{l} \psi_{1}^{\varepsilon}(x) \mathcal{X}(x, 0) \mathrm{d} x \\
& \int_{0}^{T} \int_{0}^{l}\left\{-\theta^{\varepsilon}(x, t) n_{t}(x, t)-r q^{\varepsilon}(x, t) n_{x}^{\varepsilon}(x, t)+m \psi_{x}^{\varepsilon} n_{t}(x, t)\right\} \mathrm{d} x \mathrm{~d} t \\
= & \int_{0}^{l}\left[\theta^{\varepsilon}(x, 0)-m \psi_{x}^{\varepsilon}(x, 0)\right] n(x, 0) \mathrm{d} x \\
& \int_{0}^{T} \int_{0}^{l}\left\{-\tau q^{\varepsilon}(x, t) y_{t}(x, t)+q^{\varepsilon}(x, t) y(x, t)+r \theta_{x}^{\varepsilon}(x, t) y(x, t)\right\} \mathrm{d} x \mathrm{~d} t \\
= & \int_{0}^{l} \tau q^{\varepsilon}(x, 0) y(x, 0) \mathrm{d} x
\end{aligned}
$$

for every $\mathcal{X} \in W^{1,1}\left(0, T ; L^{2}(0, l)\right) \cap L^{2}(0, T ; \mathcal{V})$ such that $\mathcal{X}(\cdot, T)=0$, for every $n \in W^{1,1}(0, T$; $\left.\left.L^{2}(0, l)\right) \cap L^{2}(0, T ; \mathcal{H})\right)$ such that $n(\cdot, T)=0$ and for every $\left.y \in W^{1,1}\left(0, T ; L^{2}(0, l)\right) \cap L^{2}(0, T ; \mathcal{V})\right)$ such that $v(\cdot, T)=0$. Next, we pass to the limit as $\varepsilon \rightarrow 0$ in the previous relations. By [5], one can obtain the relations

$$
\lim _{\varepsilon \rightarrow 0} \sup \int_{0}^{T} \int_{0}^{l}\left\{\rho_{1}\left|\varphi_{t}^{\varepsilon}(x, t)\right|^{2}-k\left[\varphi_{x}^{\varepsilon}(x, t)+\psi^{\varepsilon}(x, t)\right] \varphi_{x}^{\varepsilon}(x, t)-\alpha \varphi^{\varepsilon}(x, t) \varphi_{t}^{\varepsilon}(x, t)\right\} \mathrm{d} x \mathrm{~d} t
$$




$$
=\int_{0}^{T} \int_{0}^{l}\left\{\rho_{1}\left|\varphi_{t}^{\varepsilon}(x, t)\right|^{2}-k\left[\varphi_{x}^{\varepsilon}(x, t)+\psi^{\varepsilon}(x, t)\right] \varphi_{x}^{\varepsilon}(x, t)-\alpha \varphi^{\varepsilon}(x, t) \varphi_{t}^{\varepsilon}(x, t)\right\} \mathrm{d} x \mathrm{~d} t,
$$

which allows us to pass to the limit in the nonlinear terms even though the convergences are only weak. Accordingly, in view of convergences (3.31) and (3.32), we recover (2.3)-(2.6). This completes the proof.

\section{Exponential decay}

In this section, we prove an exponentially stability result of system (1.1)-(1.6). We introduce the following Lyapunov functional:

$$
L^{\varepsilon}(t)=\mathcal{E}^{\varepsilon}(t)+\delta_{1} I_{1}^{\varepsilon}(t)+\delta_{2} I_{2}^{\varepsilon}(t)+\delta_{3} I_{3}^{\varepsilon}(t)
$$

where

$$
\begin{aligned}
I_{1}^{\varepsilon}(t)=\int_{0}^{l}\left[\rho_{1} \varphi_{t}^{\varepsilon}(x, t) \varphi^{\varepsilon}(x, t)\right. & \left.+\rho_{2} \psi_{t}^{\varepsilon}(x, t) \psi^{\varepsilon}(x, t)\right] \mathrm{d} x+\frac{\alpha}{2} \int_{0}^{t}\left|\varphi^{\varepsilon}(x, t)\right|^{2} \mathrm{~d} x+\frac{\varepsilon}{2}\left|\varphi^{\varepsilon}(l, t)\right|^{2}, \\
I_{2}^{\varepsilon}(t) & =-\int_{0}^{l} \rho_{2}\left[\int_{0}^{x} \theta^{\varepsilon}(y, t) \mathrm{d} y\right] \psi_{t}^{\varepsilon}(x, t) \mathrm{d} x, \\
I_{3}^{\varepsilon}(t) & =-\int_{0}^{l} \tau\left[\int_{0}^{x} \theta^{\varepsilon}(y, t) \mathrm{d} y\right] q^{\varepsilon}(x, t) \mathrm{d} x,
\end{aligned}
$$

for $\delta_{1}, \delta_{2}, \delta_{3}$ are positive constants which will be fixed later.

It is easy to check that, by using Young's inequality, Poincaré's inequality and Sobolev embedding theorem, there exist two constants $\beta_{1}$ and $\beta_{2}$ such that

$$
\beta_{1} \mathcal{E}^{\varepsilon}(t) \leq L^{\varepsilon}(t) \leq \beta_{2} \mathcal{E}^{\varepsilon}(t)
$$

Next, we estimate the derivative of $L^{\varepsilon}(t)$ according to the following lemmas.

Lemma 4.1 Let $\left(\varphi^{\varepsilon}, \psi^{\varepsilon}, \theta^{\varepsilon}, q^{\varepsilon}\right)$ be the solution provided by Proposition 3.1. Then there holds

$$
\begin{aligned}
\frac{\mathrm{d}}{\mathrm{d} t} I_{1}^{\varepsilon}(t) \leq & \rho_{1} \int_{0}^{l}\left|\varphi_{t}^{\varepsilon}(x, t)\right|^{2} \mathrm{~d} x+\rho_{2} \int_{0}^{l}\left|\psi_{t}^{\varepsilon}(x, t)\right|^{2} \mathrm{~d} x-k \int_{0}^{l}\left|\varphi_{x}^{\varepsilon}(x, t)+\psi^{\varepsilon}(x, t)\right|^{2} \mathrm{~d} x \\
& -\left(b-m \eta_{1}\right) \int_{0}^{l}\left|\psi_{x}^{\varepsilon}(x, t)\right|^{2} \mathrm{~d} x-2 J^{\varepsilon}(t)+\frac{m}{4 \eta_{1}} \int_{0}^{l}\left|\theta^{\varepsilon}(x, t)\right|^{2} \mathrm{~d} x,
\end{aligned}
$$

where $C_{p}$ is a Poincaré constant, $J^{\varepsilon}(t)$ is defined in (3.7) and $\eta_{1}$ is a positive constant to be chosen later.

Proof. By differentiating (4.2) with respect to $t$ and by means of equation (3.2), we have

$$
\frac{\mathrm{d}}{\mathrm{d} t}\left\{\int_{0}^{l}\left[\rho_{1} \varphi_{t}^{\varepsilon}(x, t) \varphi^{\varepsilon}(x, t)+\rho_{2} \psi_{t}^{\varepsilon}(x, t) \psi^{\varepsilon}(x, t)\right] \mathrm{d} x\right\}
$$




$$
\begin{aligned}
= & \rho_{1} \int_{0}^{l}\left|\varphi_{t}^{\varepsilon}(x, t)\right|^{2} \mathrm{~d} x+k \int_{0}^{l}\left[\varphi_{x}^{\varepsilon}(x, t)+\psi^{\varepsilon}(x, t)\right]_{x} \varphi^{\varepsilon}(x, t) \mathrm{d} x-\frac{\alpha}{2} \frac{\mathrm{d}}{\mathrm{d} t} \int_{0}^{l}\left|\varphi^{\varepsilon}(x, t)\right|^{2} \mathrm{~d} x \\
& +\rho_{2} \int_{0}^{l}\left|\psi_{t}^{\varepsilon}(x, t)\right|^{2} \mathrm{~d} x+b \int_{0}^{l} \psi_{x x}^{\varepsilon}(x, t) \psi^{\varepsilon}(x, t) \mathrm{d} x-k \int_{0}^{l}\left[\varphi_{x}^{\varepsilon}(x, t)+\psi^{\varepsilon}(x, t)\right] \psi^{\varepsilon}(x, t) \mathrm{d} x \\
& +m \int_{0}^{l} \theta_{x}^{\varepsilon}(x, t) \psi^{\varepsilon}(x, t) \mathrm{d} x .
\end{aligned}
$$

By Hölder's inequality and Young's inequality, we get

$$
\begin{aligned}
& \frac{\mathrm{d}}{\mathrm{d} t}\left\{\int_{0}^{l}\left[\rho_{1} \varphi_{t}^{\varepsilon}(x, t) \varphi^{\varepsilon}(x, t)+\rho_{2} \psi_{t}^{\varepsilon}(x, t) \psi^{\varepsilon}(x, t)\right] \mathrm{d} x+\frac{\alpha}{2} \int_{0}^{l}\left|\varphi^{\varepsilon}(x, t)\right|^{2} \mathrm{~d} x\right\} \\
= & \rho_{1} \int_{0}^{l}\left|\varphi_{t}^{\varepsilon}(x, t)\right|^{2} \mathrm{~d} x-k \int_{0}^{l}\left[\varphi_{x}^{\varepsilon}(x, t)+\psi^{\varepsilon}(x, t)\right]^{2} \mathrm{~d} x+\rho_{2} \int_{0}^{l}\left|\psi_{t}^{\varepsilon}(x, t)\right|^{2} \mathrm{~d} x \\
& -b \int_{0}^{l}\left|\psi_{x}^{\varepsilon}(x, t)\right|^{2} \mathrm{~d} x+m \int_{0}^{l} \theta_{x}^{\varepsilon}(x, t) \psi^{\varepsilon}(x, t) \mathrm{d} x+\sigma^{\varepsilon}(t) \varphi^{\varepsilon}(l, t) \\
\leq & \rho_{1} \int_{0}^{l}\left|\varphi_{t}^{\varepsilon}(x, t)\right|^{2} \mathrm{~d} x-k \int_{0}^{l}\left[\varphi_{x}^{\varepsilon}(x, t)+\psi^{\varepsilon}(x, t)\right]^{2} \mathrm{~d} x+\rho_{2} \int_{0}^{l}\left|\psi_{t}^{\varepsilon}(x, t)\right|^{2} \mathrm{~d} x \\
& -\left(b-m \eta_{1}\right) \int_{0}^{l}\left|\psi_{x}^{\varepsilon}(x, t)\right|^{2} \mathrm{~d} x+\frac{m}{4 \eta_{1}} \int_{0}^{l}\left|\theta^{\varepsilon}(x, t)\right|^{2} \mathrm{~d} x+\tilde{\sigma}^{\varepsilon}(t) \varphi^{\varepsilon}(l, t) .
\end{aligned}
$$

For the last term on the right-hand side of (4.7), we can obtain

$$
\begin{aligned}
\tilde{\sigma}^{\varepsilon}(t) \varphi^{\varepsilon}(l, t)= & -\frac{1}{\varepsilon}\left\{\left[\varphi^{\varepsilon}(l, t)-g_{1}\right]^{+}-\left[-\varphi^{\varepsilon}(l, t)-g_{2}\right]^{+}\right\} \varphi^{\varepsilon}(l, t)-\varepsilon \varphi^{\varepsilon}(l, t) \varphi_{t}^{\varepsilon}(l, t) \\
\leq & -\frac{1}{\varepsilon}\left[\varphi^{\varepsilon}(l, t)-g_{1}\right]^{+}\left[\varphi^{\varepsilon}(l, t)-g_{1}\right]+\frac{1}{\varepsilon}\left[-\varphi^{\varepsilon}(l, t)-g_{2}\right]^{+}\left[\varphi^{\varepsilon}(l, t)+g_{2}\right] \\
& -\frac{\varepsilon}{2} \frac{\mathrm{d}}{\mathrm{d} t}\left|\varphi^{\varepsilon}(l, t)\right|^{2} \\
\leq & -\frac{1}{\varepsilon}\left[\left|\left[\varphi^{\varepsilon}(l, t)-g_{1}\right]^{+}\right|^{2}+\left|\left[-\varphi^{\varepsilon}(l, t)-g_{2}\right]^{+}\right|^{2}\right]-\frac{\varepsilon}{2} \frac{\mathrm{d}}{\mathrm{d} t}\left|\varphi^{\varepsilon}(l, t)\right|^{2} \\
= & -2 J^{\varepsilon}(t)-\frac{\varepsilon}{2} \frac{\mathrm{d}}{\mathrm{d} t}\left|\varphi^{\varepsilon}(l, t)\right|^{2} .
\end{aligned}
$$

Substituting into the previous inequality, we reach the conclusion.

Lemma 4.2 Let $\left(\varphi^{\varepsilon}, \psi^{\varepsilon}, \theta^{\varepsilon}, q^{\varepsilon}\right)$ be the solution provided by Proposition 3.1. Then there holds

$$
\begin{aligned}
\frac{\mathrm{d}}{\mathrm{d} t} I_{2}^{\varepsilon}(t) \leq & -\frac{m \rho_{2}}{2} \int_{0}^{l}\left|\psi_{t}^{\varepsilon}(x, t)\right|^{2} \mathrm{~d} x+k \eta_{2} \int_{0}^{l}\left|\varphi_{x}^{\varepsilon}(x, t)+\psi_{x}^{\varepsilon}(x, t)\right|^{2} \mathrm{~d} x \\
& +b \eta_{2} \int_{0}^{l}\left|\psi_{x}^{\varepsilon}(x, t)\right|^{2} \mathrm{~d} x+\frac{r^{2} \rho_{2}}{2 m} \int_{0}^{l}\left|q^{\varepsilon}(x, t)\right|^{2} \mathrm{~d} x \\
& +\left[\frac{b}{4 \eta_{2}}+\frac{l k}{4 \eta_{2}}+m\right] \int_{0}^{l}\left|\theta^{\varepsilon}(x, t)\right|^{2} \mathrm{~d} x
\end{aligned}
$$

for a positive constant $\eta_{2}$ to be chosen later.

Proof. By using (3.2)-(3.3) and (3.5), we find

$$
\frac{\mathrm{d}}{\mathrm{d} t} I_{2}^{\varepsilon}(t)=-\rho_{2} \int_{0}^{l}\left[\int_{0}^{x} \theta_{t}^{\varepsilon}(y, t) \mathrm{d} y\right] \psi_{t}^{\varepsilon}(x, t) \mathrm{d} x-\rho_{2} \int_{0}^{l}\left[\int_{0}^{x} \theta^{\varepsilon}(y, t) \mathrm{d} y\right] \psi_{t t}^{\varepsilon}(x, t) \mathrm{d} x
$$




$$
\begin{aligned}
= & \rho_{2} \int_{0}^{l}\left[\int_{0}^{x} r q_{y}^{\varepsilon}(y, t) \mathrm{d} y-\int_{0}^{x} m \psi_{y t}(y, t) \mathrm{d} y\right] \psi_{t}^{\varepsilon}(x, t) \mathrm{d} x-b \int_{0}^{l}\left[\int_{0}^{x} \theta^{\varepsilon}(y, t) \mathrm{d} y\right] \psi_{x x}^{\varepsilon}(x, t) \mathrm{d} x \\
& +k \int_{0}^{l}\left[\int_{0}^{x} \theta^{\varepsilon}(y, t) \mathrm{d} y\right]\left[\varphi_{x}^{\varepsilon}(x, t)+\psi^{\varepsilon}(x, t)\right] \mathrm{d} x-m \int_{0}^{l}\left[\int_{0}^{x} \theta^{\varepsilon}(y, t)\right] \theta_{x}^{\varepsilon}(x, t) \mathrm{d} x .
\end{aligned}
$$

We now estimate the right-hand side of (4.9). For a positive constant $\eta_{2}$, by Young's inequality, we get

$$
\begin{aligned}
& \rho_{2} \int_{0}^{l}\left[\int_{0}^{x} r q_{y}^{\varepsilon}(y, t) \mathrm{d} y-\int_{0}^{x} m \psi_{y t}(y, t) \mathrm{d} y\right] \psi_{t}^{\varepsilon}(x, t) \mathrm{d} x \\
= & r \rho_{2} \int_{0}^{l}\left[\int_{0}^{x} q_{y}^{\varepsilon}(y, t) \mathrm{d} y\right] \psi_{t}^{\varepsilon}(x, t) \mathrm{d} x-m \rho_{2} \int_{0}^{l}\left[\int_{0}^{x} \psi_{y t}(y, t) \mathrm{d} y\right] \psi_{t}^{\varepsilon}(x, t) \mathrm{d} x \\
\leq & \frac{r^{2} \rho_{2}}{2 m} \int_{0}^{l}\left|q^{\varepsilon}(x, t)\right|^{2} \mathrm{~d} x+\frac{m \rho_{2}}{2} \int_{0}^{l}\left|\psi_{t}^{\varepsilon}(x, t)\right|^{2} \mathrm{~d} x-m \rho_{2} \int_{0}^{l}\left|\psi_{t}^{\varepsilon}(x, t)\right|^{2} \mathrm{~d} x .
\end{aligned}
$$

By Hölder's inequality, Young's inequality, Poincaré's inequality and (3.4), (3.5), we have

$$
\begin{gathered}
-b \int_{0}^{l}\left[\int_{0}^{x} \theta^{\varepsilon}(y, t) \mathrm{d} y\right] \psi_{x x}^{\varepsilon}(x, t) \mathrm{d} x \leq b \eta_{2} \int_{0}^{l}\left|\psi_{x}^{\varepsilon}(x, t)\right|^{2} \mathrm{~d} x+\frac{b}{4 \eta_{2}} \int_{0}^{l}\left|\theta^{\varepsilon}(x, t)\right|^{2} \mathrm{~d} x, \\
k \int_{0}^{l}\left[\int_{0}^{x} \theta^{\varepsilon}(y, t) \mathrm{d} y\right]\left[\varphi_{x}^{\varepsilon}(x, t)+\psi^{\varepsilon}(x, t)\right] \mathrm{d} x \leq k \eta_{2} \int_{0}^{l}\left|\varphi_{x}^{\varepsilon}(x, t)+\psi^{\varepsilon}(x, t)\right|^{2} \mathrm{~d} x \\
+\frac{l k}{4 \eta_{2}} \int_{0}^{l}\left|\theta^{\varepsilon}(x, t)\right|^{2} \mathrm{~d} x, \\
-m \int_{0}^{l}\left[\int_{0}^{x} \theta^{\varepsilon}(y, t) \mathrm{d} y\right] \theta_{x}^{\varepsilon} \mathrm{d} x=m \int_{0}^{l}\left|\theta^{\varepsilon}(x, t)\right|^{2} \mathrm{~d} x .
\end{gathered}
$$

Combining (4.9)-(4.13), we arrive at (4.8).

Lemma 4.3 Let $\left(\varphi^{\varepsilon}, \psi^{\varepsilon}, \theta^{\varepsilon}, q^{\varepsilon}\right)$ be the solution provided by Proposition 3.1. Then there holds

$$
\begin{aligned}
\frac{\mathrm{d}}{\mathrm{d} t} I_{3}^{\varepsilon}(t) \leq & -\left[r-\eta_{3} l\right] \int_{0}^{l}\left|\theta^{\varepsilon}(x, t)\right|^{2} \mathrm{~d} x+\left[r \tau+\frac{m \tau}{4 \eta_{3}}+\frac{1}{4 \eta_{3}}\right] \int_{0}^{l}\left|q^{\varepsilon}(x, t)\right|^{2} \mathrm{~d} x \\
& +m \tau \eta_{3} \int_{0}^{l}\left|\psi_{t}^{\varepsilon}(x, t)\right|^{2} \mathrm{~d} x
\end{aligned}
$$

for a positive constant $\eta_{3}$ to be chosen later.

Proof. By using (3.2)-(3.3) and (3.5), we find

$$
\begin{aligned}
\frac{\mathrm{d}}{\mathrm{d} t} I_{3}^{\varepsilon}(t)= & -\int_{0}^{l} \tau\left[\int_{0}^{x} \theta_{t}^{\varepsilon}(y, t) \mathrm{d} y\right] q^{\varepsilon}(x, t) \mathrm{d} x-\int_{0}^{l} \tau\left[\int_{0}^{x} \theta^{\varepsilon}(y, t) \mathrm{d} y\right] q_{t}^{\varepsilon}(x, t) \mathrm{d} x \\
= & -\int_{0}^{l} \tau\left[-\int_{0}^{x} r q_{x}^{\varepsilon}(y, t) \mathrm{d} y+\int_{0}^{x} m \psi_{x t}^{\varepsilon}(y, t) \mathrm{d} y\right] q^{\varepsilon}(x, t) \mathrm{d} x \\
& -\int_{0}^{l}\left[\int_{0}^{x} \theta^{\varepsilon}(y, t) \mathrm{d} y\right]\left[-q^{\varepsilon}(x, t)-r \theta_{x}^{\varepsilon}(x, t)\right] \mathrm{d} x .
\end{aligned}
$$


Integrating by parts, we obtain

$$
\begin{aligned}
\frac{\mathrm{d}}{\mathrm{d} t} I_{3}^{\varepsilon}(t)= & r \tau \int_{0}^{l}\left|q^{\varepsilon}(x, t)\right|^{2} \mathrm{~d} x-m \tau \int_{0}^{l} \psi_{t}^{\varepsilon}(x, t) q^{\varepsilon}(x, t) \mathrm{d} x+\int_{0}^{l}\left[\int_{0}^{x} \theta^{\varepsilon}(y, t) \mathrm{d} y\right] q^{\varepsilon}(x, t) \mathrm{d} x \\
& -r \int_{0}^{l}\left|\theta^{\varepsilon}(x, t)\right|^{2} \mathrm{~d} x .
\end{aligned}
$$

By means of Hölder's inequality, Young's inequality, Poincaré's inequality, we deduce (4.14).

Proof of Theorem 2.3. From (3.28), (4.6), (4.8) and (4.14), then from (4.1), we obtain

$$
\begin{aligned}
\frac{\mathrm{d}}{\mathrm{d} t} L^{\varepsilon}(t) \leq & -\left[\alpha-\delta_{1} \rho_{1}\right] \int_{0}^{l}\left|\varphi_{t}^{\varepsilon}(x, t)\right|^{2} \mathrm{~d} x-\left[\frac{\delta_{2} m \rho_{2}}{2}-\delta_{1} \rho_{2}-\delta_{3} m \tau \eta_{3}\right] \int_{0}^{l}\left|\psi_{t}^{\varepsilon}(x, t)\right|^{2} \mathrm{~d} x \\
& -k\left[\delta_{1}-\delta_{2} \eta_{2}\right] \int_{0}^{l}\left|\varphi_{x}^{\varepsilon}(x, t)+\psi^{\varepsilon}(x, t)\right|^{2} \mathrm{~d} x-\left[\delta_{1}\left(b-m \eta_{1}\right)-\delta_{2} b \eta_{2}\right] \int_{0}^{l}\left|\psi_{x}^{\varepsilon}(x, t)\right|^{2} \mathrm{~d} x \\
& -\left[\delta_{3}\left(r-\eta_{3} l\right)-\frac{\delta_{1} m}{4 \eta_{1}}-\delta_{2}\left(\frac{b}{4 \eta_{2}}+\frac{l k}{4 \eta_{2}}+m\right)\right] \int_{0}^{l}\left|\theta^{\varepsilon}(x, t)\right|^{2} \mathrm{~d} x \\
& -\left[1-\frac{\delta_{2} r^{2} \rho_{2}}{2 m}-\delta_{3}\left(r \tau+\frac{m \tau}{4 \eta_{3}}+\frac{1}{4 \eta_{3}}\right)\right] \int_{0}^{l}\left|q^{\varepsilon}(x, t)\right|^{2} \mathrm{~d} x-2 \delta_{1} J^{\varepsilon}(t)-\varepsilon\left|\varphi_{t}^{\varepsilon}(l, t)\right|^{2} .
\end{aligned}
$$

In fact, we first choose $\eta_{1}<\frac{b}{2 m}, \eta_{3}<\frac{r}{2 l}$ and $\delta_{2}<\frac{m}{r^{2} \rho_{2}}$ small enough so that

$$
\left\{\begin{array}{l}
b-m \eta_{1}>0 \\
r-\eta_{3} l>0 \\
1-\frac{\delta_{2} r^{2} \rho_{2}}{2 m}>0
\end{array}\right.
$$

By choosing $\delta_{3}<\frac{1}{2\left(r \tau+\frac{m \tau}{4 \eta_{3}}+\frac{1}{4 \eta_{3}}\right)}$ small enough, we have

$$
1-\frac{\delta_{2} r^{2} \rho_{2}}{2 m}-\delta_{3}\left(r \tau+\frac{m \tau}{4 \eta_{3}}+\frac{1}{4 \eta_{3}}\right)>0 .
$$

Next, we take $\delta_{1}<\frac{\alpha}{\rho_{1}}$ and $\eta_{2}<\frac{\delta_{1}}{2 \delta_{2}}$ such that

$$
\left\{\begin{array}{l}
\alpha-\delta_{1} \rho_{1}>0 \\
\delta_{1}-\delta_{2} \eta_{2}>0 \\
\delta_{1}\left(b-m \eta_{1}\right)-\delta_{2} b \eta_{2}>0
\end{array}\right.
$$

Once $\delta_{2}, \delta_{3}$ are fixed, we take $\delta_{1}<\min \left\{\frac{\delta_{2} m \rho_{2}}{2 \rho_{2}}-\frac{\delta_{3} m \tau \eta_{3}}{\rho_{2}}, \frac{4 \eta_{1} \delta_{3}}{m}\left(r-\eta_{3} l\right)-\frac{4 \eta_{1} \delta_{2}}{m}\left(\frac{b}{4 \eta_{2}}+\frac{l k}{4 \eta_{2}}+m\right)\right\}$ so that

$$
\left\{\begin{array}{l}
\frac{\delta_{2} m \rho_{2}}{2}-\delta_{1} \rho_{2}-\delta_{3} m \tau \eta_{3}>0 \\
\delta_{3}\left(r-\eta_{3} l\right)-\frac{\delta_{1} m}{4 \eta_{1}}-\delta_{2}\left(\frac{b}{4 \eta_{2}}+\frac{l k}{4 \eta_{2}}+m\right)>0
\end{array}\right.
$$

combined with $\delta_{1}<\frac{\alpha}{\rho_{1}}$, we can obtain

$$
\delta_{1}<\min \left\{\frac{\alpha}{\rho_{1}}, \frac{\delta_{2} m \rho_{2}}{2 \rho_{2}}-\frac{\delta_{3} m \tau \eta_{3}}{\rho_{2}}, \frac{4 \eta_{1} \delta_{3}}{m}\left(r-\eta_{3} l\right)-\frac{4 \eta_{1} \delta_{2}}{m}\left(\frac{b}{4 \eta_{2}}+\frac{l k}{4 \eta_{2}}+m\right)\right\} .
$$


Hence, We infer the estimate

$$
\frac{\mathrm{d}}{\mathrm{d} t} L^{\varepsilon}(t) \leq-C_{0} \mathcal{E}^{\varepsilon}(t) \leq-\frac{C_{0}}{\beta_{2}} L^{\varepsilon}(t)
$$

with a positive constant $C_{0}$. By direct integration over $\left(t_{0}, t\right)$, we have

$$
L^{\varepsilon}(t) \leq L^{\varepsilon}(0) e^{-\frac{C_{0}}{\beta_{2}} t}
$$

which, combined with (4.5) with $M=\frac{C_{2}}{\beta_{1}}$ and $\gamma=\frac{C_{0}}{\beta_{2}}$, we can obtain

$$
\mathcal{E}^{\varepsilon}(t) \leq M \mathcal{E}^{\varepsilon}(0) e^{-\gamma t}
$$

By (3.1), we get

$$
J^{\varepsilon}(0)=\frac{1}{2 \varepsilon}\left\{\left|\left[\varphi^{\varepsilon}(l, 0)-g_{1}\right]^{+}\right|^{2}+\left|\left[-\varphi^{\varepsilon}(l, 0)\right]^{+}\right|^{2}\right\}=0 .
$$

Accordingly, we have $\mathcal{E}^{\varepsilon}(0)=J^{\varepsilon}(0)+E^{\varepsilon}(0)=E^{\varepsilon}(0)$. In view of (4.15), the inequality

$$
E^{\varepsilon}(t) \leq \mathcal{E}^{\varepsilon}(t) \leq M \mathcal{E}^{\varepsilon}(0) e^{-\gamma t}=M E^{\varepsilon}(0) e^{-\gamma t}
$$

holds. By passing to $\lim _{\varepsilon \rightarrow 0}$ inf and on account of (3.27) and (3.31), we reach the conclusion.

\section{Acknowledgments}

This work was supported by the National Natural Science Foundation of China [grant number 11301277], the Natural Science Foundation of Jiangsu Province [grant number BK20151523], the Six Talent Peaks Project in Jiangsu Province [grant number 2015-XCL-020] and the Qing Lan Project of Jiangsu Province.

\section{References}

[1] F. Alabau-Boussouira, Asymptotic behavior for Timoshenko beams subject to a single nonlinear feedback control, NoDEA Nonlinear Differential Equations Appl. 14 (2007), no. 5-6, 643-669.

[2] K. T. Andrews, M. Shillor and S. Wright, On the dynamic vibrations of an elastic beam in frictional contact with a rigid obstacle, J. Elasticity 42 (1996), no. 1, 1-30.

[3] H. Antes and P. D. Panagiotopoulos, The boundary integral approach to static and dynamic contact problems, International Series of Numerical Mathematics, 108, Birkhäuser, Basel, 1992.

[4] S. Arantes, J. Rivera, Exponential decay for a thermoelastic beam between two stops, J. Journal of Thermal Stresses 31 (2008), no. 6, 537-556.

[5] F. D. Araruna, A. J. R. Feitosa and M. L. Oliveira, A boundary obstacle problem for the Mindlin-Timoshenko system, Math. Methods Appl. Sci. 32 (2009), no. 6, 738-756. 
[6] A. Berti et al., Analysis of dynamic nonlinear thermoviscoelastic beam problems, Nonlinear Anal. 95 (2014), 774-795.

[7] A. Berti, J. E. MuMuMuoz Rivera and M. G. Naso, A contact problem for a thermoelastic Timoshenko beam, Z. Angew. Math. Phys. 66 (2015), no. 4, 1969-1986.

[8] A. Berti et al., A dynamic thermoviscoelastic contact problem with the second sound effect, J. Math. Anal. Appl. 421 (2015), no. 2, 1163-1195.

[9] A. Berti and M. G. Naso, Vibrations of a damped extensible beam between two stops, Evol. Equ. Control Theory 2 (2013), no. 1, 35-54.

[10] F. Boulanouar and S. Drabla, General boundary stabilization result of memory-type thermoelasticity with second sound, Electron. J. Differential Equations 2014 (2014), No. 202, 18 pp.

[11] D. E. Carlson, Linear Thermoelasticity, in: Handbuch der Physik, Vol.VIa/2, edited by C. Truesdell, Spinger, Berlin, 1972.

[12] M. M. Cavalcanti et al., Uniform decay rates for the energy of Timoshenko system with the arbitrary speeds of propagation and localized nonlinear damping, Z. Angew. Math. Phys. 65 (2014), no. 6, 1189-1206.

[13] M. M. Chen, W. J. Liu and W. C. Zhou, Existence and general stabilization of the Timoshenko system of thermo-viscoelasticity of type III with frictional damping and delay terms, Adv. Nonlinear Anal., Online First. DOI: 10.1515/anona-2016-0085

[14] M. I. M. Copetti, Numerical approximation of dynamic deformations of a thermoviscoelastic rod against an elastic obstacle, M2AN Math. Model. Numer. Anal. 38 (2004), no. 4, 691-706.

[15] M. I. M. Copetti, A contact problem in generalized thermoelasticity, Appl. Math. Comput. 218 (2011), no. 5, 2128-2145.

[16] M. I. M. Copetti and J. R. Fernández, A dynamic contact problem involving a Timoshenko beam model, Appl. Numer. Math. 63 (2013), 117-128.

[17] W. A. Day, Heat Conduction within Linear Thermoelasticity, 3rd Edition, Pergamon, Oxford, 1986.

[18] A. Djebabla and N. Tatar, Exponential stabilization of the Timoshenko system by a thermoviscoelastic damping, J. Dyn. Control Syst. 16 (2010), no. 2, 189-210.

[19] A. Djebabla and N. Tatar, Stabilization of the Timoshenko beam by thermal effect, Mediterr. J. Math. 7 (2010), no. 3, 373-385. 
[20] G. Duvaut and J.-L. Lions, Inequalities in mechanics and physics, translated from the French by C. W. John, Springer, Berlin, 1976.

[21] C. M. Elliott and Q. Tang, A dynamic contact problem in thermoelasticity, Nonlinear Anal. 23 (1994), no. 7, 883-898.

[22] H. D. Fernández Sare and R. Racke, On the stability of damped Timoshenko systems: Cattaneo versus Fourier law, Arch. Ration. Mech. Anal. 194 (2009), no. 1, 221-251.

[23] A. E. Green and K. A. Lindsay, Thermoelasticity, J. Elasticity, 2 (1972), 1-7.

[24] J.-R. Kang, Asymptotic behavior of the thermoelastic suspension bridge equation with linear memory, Bound. Value Probl. 2016 (2016), no. 206, 18 pp.

[25] J. U. Kim and Y. Renardy, Boundary control of the Timoshenko beam, SIAM J. Control Optim. 25 (1987), no. 6, 1417-1429.

[26] K. L. Kuttler and M. Shillor, A dynamic contact problem in one-dimensional thermoviscoelasticity, Nonlinear World 2 (1995), no. 3, 355-385.

[27] K. L. Kuttler and M. Shillor, Vibrations of a beam between two stops, Dyn. Contin. Discrete Impuls. Syst. Ser. B Appl. Algorithms 8 (2001), no. 1, 93-110.

[28] W. J. Liu, K. W. Chen and J. Yu, Existence and general decay for the full von Karman beam with a thermo-viscoelastic damping, frictional dampings and a delay term, IMA J. Math. Control Inform., Online First. DOI:10.1093/imamci/dnv056 cite:1+2

[29] W. J. Liu and M. M. Chen, Well-posedness and exponential decay for a porous thermoelastic system with second sound and a time-varying delay term in the internal feedback, Contin. Mech. Thermodyn., Online First. DOI: 10.1007/s00161-017-0556-z

[30] H. W. Lord and Y. Shulman, A generalized dynamical theory of thermoelasticity, J. Mech. Phys. Sol. 15 (1967), 299-309.

[31] S. A. Messaoudi and A. Fareh, Energy decay in a Timoshenko-type system of thermoelasticity of type III with different wave-propagation speeds, Arab. J. Math. (Springer) 2 (2013), no. 2, 199-207.

[32] S. A. Messaoudi and B. Said-Houari, Exponential stability in one-dimensional non-linear thermoelasticity with second sound, Math. Methods Appl. Sci. 28 (2005), no. 2, 205-232.

[33] S. A. Messaoudi and B. Said-Houari, Energy decay in a Timoshenko-type system of thermoelasticity of type III, J. Math. Anal. Appl. 348 (2008), no. 1, 298-307.

[34] J. E. Muñoz Rivera and M. de Lacerda Oliveira, Exponential stability for a contact problem in thermoelasticity, IMA J. Appl. Math. 58 (1997), no. 1, 71-82. 
[35] J. E. Muñoz Rivera and R. Racke, Global stability for damped Timoshenko systems, Discrete Contin. Dyn. Syst. 9 (2003), no. 6, 1625-1639.

[36] Y. M. Qin and Z. Y. Ma, Global well-posedness and asymptotic behavior of the solutions to non-classical thermo(visco)elastic models, Springer, Singapore (2016).

[37] R. Racke, Thermoelasticity with second sound - exponential stability in linear and non-linear 1-d, Math. Methods Appl. Sci. 25 (2002), no. 5, 409-441.

[38] R. Racke, Asymptotic behavior of solutions in linear 2- or 3-D thermoelasticity with second sound, Quart. Appl. Math. 61 (2003), no. 2, 315-328.

[39] R. Racke and Y.-G. Wang, Asymptotic behavior of discontinuous solutions to thermoelastic systems with second sound, Z. Anal. Anwendungen 24 (2005), no. 1, 117-135.

[40] C. A. Raposo et al., Exponential stability for the Timoshenko system with two weak dampings, Appl. Math. Lett. 18 (2005), no. 5, 535-541.

[41] B. Said-Houari and A. Kasimov, Decay property of Timoshenko system in thermoelasticity, Math. Methods Appl. Sci. 35 (2012), no. 3, 314-333.

[42] P. Shi and M. Shillor, Uniqueness and stability of the solution to a thermoelastic contact problem, European J. Appl. Math. 1 (1990), no. 4, 371-387.

[43] A. Soufyane, Exponential stability of the linearized nonuniform Timoshenko beam, Nonlinear Anal. Real World Appl. 10 (2009), no. 2, 1016-1020.

[44] F. Tahamtani, On energy decay of an $n$-dimensional thermoelasticity system with a nonlinear weak damping, Iran. J. Sci. Technol. Trans. A Sci. 32 (2008), no. 1, 45-51.

[45] M. A. Tarabek, On the existence of smooth solutions in one-dimensional nonlinear thermoelasticity with second sound, Quart. Appl. Math. 50 (1992), no. 4, 727-742.

[46] Q.-X. Yan, S.-H. Hou and D.-X. Feng, Asymptotic behavior of Timoshenko beam with dissipative boundary feedback, J. Math. Anal. Appl. 269 (2002), no. 2, 556-577.

(C) 2017 by the authors; licensee Preprints, Basel, Switzerland. This article is an open access article distributed under the terms and conditions of the Creative Commons Attribution (CC BY) license http://creativecommons.org/licenses/by/4.0/). 